\section{More on hypertension and reduced renal function}

\author{
M. La Regina • C. del Prato
}

\author{
M. La Regina $(\bowtie) \cdot$ C. del Prato \\ U.O.C Pronto Soccorso e Medicina d'Urgenza \\ Ospedale Civile Sant'Andrea, \\ Via V. Veneto 197, I-19124 La Spezia, Italy \\ e-mail: micaela.laregina@nafura.it
}

Received: 8 August 2006 / Accepted in original form: 30 August 2006 / Published online: 31 March 2007

First of all, compliments for the new and wider "dress and mission" of the journal of our society. As it is destined for a double audience, it is no surprise that each paper will naturally be seen from two different points of view. Even though many of the Italian emergency physicians (EP), at the beginning of Italian speciality courses in emergency medicine (EM), started out in Internal Medicine, as they have been working in EM they have changed quickly their clinical style because of unstable clinical conditions of some patients, and more pressing rhythms that have to be faced. One must develop a quicker, simpler and more pragmatic way of thinking and acting: does the patient have a life-threatening disease or not? Does the patient need hospitalisation or is it safe for a primary care physician (PCP) to manage the problem on an outpatient basis? The introduction of cost/benefit ratios in medicine is creating another pressure: not to admit the patient.

As internists we read with great interest the case report in the first issue [1], but as physicians working in EM, we immediately transferred its contents to our practice. How many patients with increased blood pressure (BP) can we "appropriately" hospitalise? Surely, the ones with a complicated hypertensive crisis, like the patient in the case report. What about the others? Is it enough to reduce the BP at presentation, re-educate them on diet and treatment compliance, and then send them to the PCP with a recommendation to keep a diary of their BP for 10-14 days? Maybe diagnosing secondary hypertension is not the responsibility of the EP, especially during the first presentation and in asymptomatic cases, but can the EP prevent, in some way, deterioration requiring further visits to the emergency department (ED) for the same level of hypertension as well as more complicated deteriorations?

The patient in this case report had already been evaluated for mild nocturnal dyspnoea and palpitations associated with an increased BP, and treated with an addition of diuretics. Blood tests and ultrasound scan 1 month before could have prevented the pulmonary oedema and the need for hospitalisation, even though it would have been more expensive than a simple introduction of diuretics.

It is obvious we cannot screen all hypertensive and dyslipidaemic patients coming to the emergency room with duplex ultrasonographic scanning, as this is not a readily available resource, nor can we undertake other examinations for other secondary kinds of hypertension, nor can we hospitalise all of them. It is true that we would not miss any diagnoses of secondary hypertension if we were able to do these things.

What, then, would be the best alternative to achieve an efficacious, safe and cost-effective practice for an EP facing an asymptomatic hypertensive patient who exhibits strong compliance with diet and treatment?

Should we test the serum creatinine and potassium levels, and hospitalise the patients with altered values? Should we hospitalise patients whose clinical presentation suggests secondary hypertension? Most hypertensive patients take diuretics, and will routinely have slightly elevated creatinine and potassium. Moreover, in many rural and peripheral small EDs, it is impossible to obtain blood tests at night!

Should we refer the patients to the PCP for the blood tests, including creatinine clearance and close follow-up? The reality is that patients are sent to us by the PCP, who often encounters obstacles in having early appointments for diagnostic and imaging procedures.

Should we refer the patients to EM outpatient clinics?

Should we refer the patients to Internal Medicine or Cardiology for same-day hospital evaluations? Formerly we used to hospitalise asymptomatic hypertensive patients with a strong possibility of secondary hypertension (a BP that remains elevated in spite of different treatments in the ED).

Should we have the patient return to the ED for brief follow-up over a short time period, especially if they have altered blood tests where available, and refer all the others to the PCP? At present, we are just starting a complex assistance package project, sustained by our regional government. It would be interesting to know about similar experiences regarding this and other diagnoses.

\section{Reference}

1. Ungar A, Lambertucci L, Agresti C et al (2006) Hypertension and reduced renal function in an 83-year-old patient. Intern Emerg Med 1:40-48

Intern Emerg Med (2007) 2:62-63

\section{Hypertension and reduced renal function: a rebuttal}

\section{P.A. Modesti • G. Parati}

P.A. Modesti ( $ه)$

Clinical Medicine and Cardioilogy Department of Critical Care Medicine and Surgery 